\title{
Biometria, razão sexual e dimorfismo sexual de Trachemys dorbigni (Duméril \& Bibron 1835) (Testudines, Emydidae) em um açude no município de São Gabriel, Rio Grande do Sul, Brasil
}

\author{
Melise Lucas Silveira ${ }^{1 *}$ \\ Marilia Teresinha Hartmann ${ }^{2}$ \\ Alex Bager ${ }^{1}$ \\ ${ }^{1}$ Departamento de Biologia, Universidade Federal de Lavras \\ Campus Universitário, CEP 37200-000, Lavras - MG, Brasil \\ ${ }^{2}$ Universidade Federal da Fronteira Sul, Campus Erechim \\ CEP 99700-000, Erechim - RS, Brasil \\ * Autor para correspondência \\ melisesilveira@hotmail.com
}

Submetido em 28/08/2011

Aceito para publicação em 10/04/2012

\section{Resumo}

Com o intuito de se estudar a estrutura de uma população de Trachemys dorbigni, foram realizadas coletas durante o período de março de 2009 a fevereiro de 2010, em um açude $\left(30^{\circ} 20^{\prime} 16,03^{\prime \prime}\right.$ S e $\left.54^{\circ} 15^{\prime} 46,68^{\prime \prime} \mathrm{W}\right)$, no município de São Gabriel, Rio Grande do Sul, Brasil. Foram coletados 44 indivíduos, sendo 34 fêmeas, sete machos e três juvenis. A razão sexual encontrada foi diferente de 1:1 (4,85 fêmeas:1 macho). O comprimento médio da carapaça dos adultos foi igual a $190,50 \pm 22,81 \mathrm{~mm}(\mathrm{~N}=41)$ e a massa média foi igual a $1,210 \pm 420 \mathrm{~g}$ $(\mathrm{N}=37)$. Os machos apresentaram comprimento médio da carapaça de $179,10 \pm 18,60 \mathrm{~mm}(\mathrm{~N}=7)$ e as fêmeas, de $192,90 \pm 23,10 \mathrm{~mm}(\mathrm{~N}=34)$. As fêmeas mostraram-se significativamente maiores do que os machos em seis medidas das onze obtidas e menores em apenas uma medida, comprimento da base da cauda ao orifício cloacal. A análise discriminante mostrou diferenças morfológicas significativas entre machos e fêmeas $(P<0,05$; $F_{3.37}=21,02$; Wilks' lambda $=0,36$ ), indicando a distância terminal entre a carapaça e o plastrão, o comprimento curvilíneo da carapaça e o comprimento da base da cauda ao orifício cloacal como variáveis importantes na diferenciação entre os sexos.

Palavras-chave: Estrutura populacional; Morfometria; Tigre d'água; Variação da forma

\section{Abstract}

Biometrics, sex ratio and sexual dimorphism of Trachemys dorbigni (Duméril \& Bibron 1835) (Testudines, Emydidae) from an artificial reservoir in the municipality of São Gabriel, Rio Grande do Sul, Brazil. This work describes the population structure of Trachemys dorbigni from an artificial reservoir $\left(30^{\circ} 20^{\prime} 16,03^{\prime \prime}\right.$, $\left.54^{\circ} 15^{\prime} 46,68^{\prime \prime} \mathrm{W}\right)$ in the municipality of São Gabriel, Rio Grande do Sul, Brazil. From March 2009 to February 2010, forty-four individuals were collected: 34 females, seven males, and three juveniles. The sex ratio was 4.85 females: 1 male (1:1). The mean carapace length of the adults was $190.50 \pm 22.81 \mathrm{~mm}(\mathrm{~N}=41)$ and the mean body mass was $1.210 \pm 420 \mathrm{~g}(\mathrm{~N}=37)$. Carapace length averaged $179.10 \pm 18.60 \mathrm{~mm}(\mathrm{~N}=7)$ for 
males and $192.90 \pm 23.10 \mathrm{~mm}(\mathrm{~N}=34)$ for females. Females were significantly larger than males for six of the eleven measurements made and smaller for one measurement, which was the distance from the base of tail to the cloacae. The discriminant analysis revealed significant morphological differences between males and females $\left(P<0,05 ; F_{3.37}=21.02\right.$; Wilks' lambda=0.36), indicating carapace and plastron terminal distance, curvilinear carapace length and distance from the base of tail to the cloacae as important variables for sexing this species.

Key words: D’Orbigny's slider turtle; Morphometry; Population structure; Shape variation

\section{Introdução}

Trachemys é o único gênero de Emydidae encontrado na América Central e do Sul (SEIDEL, 2002). Conforme Seidel (2002), Trachemys é representado atualmente por 15 espécies, mas se reconhecem 26 formas diferentes que podem ser consideradas subespécies, dependendo dos critérios adotados. No Brasil, o gênero Trachemys está representado por duas espécies: T. adiutrix (VANZOLINI, 1995), restrita ao estado do Maranhão e Piauí (BATISTELLA et al., 2008), e T. dorbigni (DUMERIL \& BIBRON, 1835), que tem sua distribuição no Rio Grande do Sul, além do norte da Argentina e Uruguai (FRITZ; HAVAS, 2007). Trachemys dorbigni é o quelônio mais abundante do Rio Grande do Sul (PEREIRA; DIEFENBACH, 2001), sofrendo diversos impactos ao seu habitat, como contaminação dos rios, destruição da mata ciliar e comercialização ilegal de filhotes como animais de estimação, atividades que influenciam na estabilidade das populações dessa espécie (MOLINA; GOMES, 1998; BUJES; VERRASTRO, 2008). Atividades agrícolas também são um impacto negativo para a conservação de T. dorbigni principalmente nas áreas de desova, onde segundo Bager e Rosado (2010), é necessária a preservação de uma faixa com $94 \mathrm{~m}$ de extensão (medidos a partir da margem dos corpos d'água) para que se proteja $90 \%$ dos seus ninhos. Neste contexto, o conhecimento de parâmetros populacionais pode fornecer subsídios ao desenvolvimento de estratégias de conservação da espécie. As descrições da estrutura etária, da razão sexual e de variações morfológicas entre os sexos são necessárias na interpretação dos processos ecológicos que atuam em uma população (GIBBONS et al., 2001; APONTE et al., 2003).

No presente estudo, caracterizamos uma população de $T$. dorbigni em uma área no interior do Rio Grande do Sul. Foram analisados: (1) morfometria e dimorfismo sexual; (2) estrutura da população através da razão sexual e das classes de tamanho.

\section{Material e Métodos}

\section{Área de estudo}

O estudo foi realizado no município de São Gabriel (30²0'9”'S, 54¹9'12’W), estado do Rio Grande do Sul, Brasil. Conforme Maluf (2000), São Gabriel apresenta clima de tipo subtemperado seco, com temperatura média anual de $18,5^{\circ} \mathrm{C}$ e temperatura média do mês mais frio de $12,5^{\circ} \mathrm{C}$, com precipitação anual de $1.355 \mathrm{~mm}$ e apresentando altitude máxima de $114 \mathrm{~m}$. As coletas foram realizadas em um açude com área aproximada de $900 \mathrm{~m}^{2}$, inserido em uma matriz de paisagem agropastoril

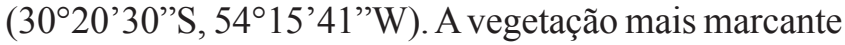
do açude é composta de macrófitas flutuantes e fixas, predominando Nymphoides indica.

\section{Coleta de dados}

Foram realizadas amostragens mensais, entre março de 2009 e fevereiro de 2010, totalizando 81 dias de amostragens. As capturas foram realizadas com quatro armadilhas "box trap", de arame galvanizado, cada uma com $600 \mathrm{~mm}$ de largura, $360 \mathrm{~mm}$ de altura e $800 \mathrm{~mm}$ de comprimento, iscadas com carne de frango. As armadilhas foram dispostas parcialmente submersas em quatro pontos diferentes no corpo d'água e verificadas duas vezes ao dia, durante três a cinco dias consecutivos. Todos os indivíduos capturados foram medidos, pesados e marcados. A marcação consistiu em um corte nos escudos marginais conforme Cagle (1939). Para a pesagem utilizou-se balança digital com capacidade para $5 \mathrm{~kg}$ e acuracidade de $0,01 \mathrm{~g}$. Para a biometria, foi utilizado um paquímetro de $300 \mathrm{~mm}$ para as medidas do casco, e outro de $150 \mathrm{~mm}$ para as medidas 
da cauda, ambos com acuracidade de $0,01 \mathrm{~mm}$, e para as medidas curvilíneas foi utilizado uma fita métrica flexível. Foram obtidos 10 registros morfométricos: 1) CMC - comprimento linear máximo da carapaça; 2) LMC - largura linear máxima da carapaça; 3) ALT altura linear máxima do casco; 4) CSMV - comprimento linear da sutura médio ventral; 5) CMP - comprimento linear máximo do plastrão; 6) LMP - largura linear máxima do plastrão, medida entre os escudos peitorais e abdominais; 7) CCD1 - comprimento linear da base da cauda ao orifício cloacal; 8) CCD2 - comprimento linear da cauda; 9) CCC - comprimento curvilíneo da carapaça; 10) VAO - distância entre o final da sutura médio ventral e os escudos supracaudais. O sexo dos adultos foi determinado com base nas seguintes características secundárias observadas nos machos (CABRERA, 1998): cauda comprida e larga na base, com cloaca em posição distal e existência do processo de melanização. Com base em estudos anteriores de Trachemys dorbigni (FAGUNDES et al., 2010), os indivíduos que apresentaram tamanho mínimo já reportado para machos e que não revelaram as características sexuais secundárias foram considerados fêmeas.

\section{Análise dos dados}

Foi efetuada uma análise de variância, usando-se o teste de Kruskal-Wallis, utilizando o programa BioEstat 5.0, a fim de verificar diferenças na estrutura de tamanho entre machos e fêmeas. Os dimorfismos sexuais foram testados a partir da análise discriminante (Backward), no programa Statistica 6. Para tanto, as medidas CMC, LMC, ALT, CSMV, CMP, LMP, CCD1, CCD2, CCC e VAO foram consideradas como variáveis independentes, aplicadas aos indivíduos adultos. Os juvenis foram desconsiderados nas análises evitando assim possíveis variações ontogenéticas que influenciassem na forma. Os dados utilizados foram testados quanto a normalidade por Shapiro-Wilk (W). Para testar a significância da razão sexual foi aplicado o teste do Qui-quadrado, disponível no programa BioEstat 5.0. Todas as análises foram realizadas com um nível de significância de 0,05 .

\section{Resultados}

Foram capturados 44 indivíduos de T. dorbigni, sendo 34 fêmeas, sete machos e três juvenis. A razão sexual foi desviada para fêmeas, sendo significativamente diferente de 1:1 (4,85 fêmeas: 1 macho) $\left(\chi^{2}=17,78 ;\right.$ g.l. =1; $P<0,05)$.

Os machos apresentaram CMC médio de 179,10 $\pm 18,60 \mathrm{~mm}(\mathrm{~N}=7)$ e as fêmeas $192,90 \pm 23,10 \mathrm{~mm}$ $(\mathrm{N}=34)$ (Tabela 1). A distribuição de frequência por classes de tamanho apresentou tendência unimodal para as fêmeas, com a maior frequência do CMC em 200mm. Para os machos a distribuição foi claramente unimodal com classe modal em 180mm (Figura 1). As

TABELA 1: Estatística descritiva de 11 variáveis medidas de exemplares de Trachemys dorbigni, capturados em São Gabriel, Rio Grande do Sul, Brasil. Todas as medidas estão em milímetros e o peso em gramas. N= número de indivíduos; $\overline{\mathrm{x}}=$ média; Mín = mínimo; Máx = máximo; $\mathrm{E} . \mathrm{P}=$ erro padrão da média; $P=$ nível de significância da análise de variância.

\begin{tabular}{lccccccccccc}
\hline & \multicolumn{3}{c}{ Machos } & \multicolumn{9}{c}{ Fêmeas } \\
\cline { 2 - 11 } & $\mathbf{N}$ & $\overline{\mathbf{x}}$ & Mín & Máx & $\mathbf{E . P}$ & $\mathbf{N}$ & $\overline{\mathbf{x}}$ & Mín. & Máx. & E.P & $\boldsymbol{P}$ \\
\hline CMC & 7 & 179,1 & 155,2 & 209,3 & 7,0 & 34 & 192,9 & 148,0 & 224,6 & 4,0 & 0,11 \\
LMC & 7 & 136,8 & 120,9 & 152,4 & 3,7 & 34 & 147,1 & 113,4 & 173,3 & 2,7 & 0,06 \\
ALT & 6 & 81,5 & 71,2 & 91,7 & 2,8 & 34 & 91,9 & 66,7 & 115,6 & 2,1 & $<0,05$ \\
CSMV & 7 & 156,4 & 129,5 & 176,9 & 6,3 & 34 & 172,3 & 98,5 & 205,8 & 4,4 & $<0,05$ \\
CMP & 7 & 163,3 & 140,9 & 184,4 & 5,7 & 34 & 180,4 & 123,7 & 211,9 & 3,8 & $<0,05$ \\
LMP & 7 & 88,6 & 80,5 & 100,5 & 2,5 & 34 & 101,0 & 78,3 & 186,2 & 3,2 & $<0,05$ \\
VAO & 7 & 25,0 & 21,4 & 30,8 & 1,1 & 34 & 23,1 & 15,1 & 28,0 & 0,5 & 0,08 \\
CCC & 7 & 202,1 & 170,0 & 235,0 & 8,8 & 34 & 224,6 & 170,0 & 280,0 & 5,1 & $<0,05$ \\
CCD1 & 7 & 17,3 & 12,0 & 22,8 & 1,3 & 34 & 12,0 & 8,6 & 16,0 & 0,3 & $<0,05$ \\
CCD2 & 7 & 38,4 & 23,7 & 44,2 & 2,7 & 34 & 37,9 & 26,4 & 49,5 & 0,9 & 0,55 \\
Peso & 7 & 927,7 & 595,0 & 1322,0 & 93,6 & 31 & 1270,0 & 550,0 & 2090,0 & 77,3 & $<0,05$ \\
\hline
\end{tabular}


fêmeas mostraram-se significativamente maiores do que os machos em seis medidas (ALT, CSMV, CMP, LMP, CCC e Peso) e menores na CCD1 (Tabela 1).

A análise discriminante mostrou que existem diferenças morfométricas significativas entre machos e fêmeas de $T$. dorbigni $\left(P<0,05 ; F_{3.37}=21,02\right.$; Wilks' lambda $=0,36)$, e que as variáveis $\mathrm{VAO}, \mathrm{CCC}$ e CCD1 são medidas importantes na diferenciação entre os sexos (Tabela 2). O nível de acerto da equação da análise discriminante foi de $97,56 \%$ (fêmeas $=100 \%$; machos $=$ $85,71 \%)$.

TABELA 2: Coeficientes canônicos da análise discriminante realizada para 41 exemplares de Trachemys dorbigni, capturados em São Gabriel, Rio Grande do Sul, Brasil. VAO= distância entre o final da sutura médio ventral e os escudos supracaudais; $\mathrm{CCC}=$ comprimento curvilíneo da carapaça; $\mathrm{CCD} 1=$ distância da base da cauda ao orifício cloacal; CAN I= coeficiente canônico.

\begin{tabular}{cc}
\hline & CAN I \\
\hline VAO & 0,904 \\
CCC & $-1,130$ \\
CCD1 & 0,827 \\
\hline
\end{tabular}

\section{Discussão}

No presente estudo, observou-se uma razão sexual desviada a favor das fêmeas. Gibbons (1990) sugere que a existência de variação da razão sexual entre populações da mesma espécie pode ser o resultado de um viés metodológico, como a utilização de diferentes métodos de captura, ou de questões ecológicas, como a exibição de um comportamento diferenciado entre os sexos. Existe a possibilidade de ocorrer diferenças na razão sexual devido a uma determinação do sexo dependente da temperatura de incubação (DST) dos ovos (DODD, 1989). Embora, não existam informações do modo de determinação do sexo em T. dorbigni, a DST ocorre para algumas espécies do gênero Trachemys, sendo bem conhecida em T. scripta (WIBBELS et al., 1998; DODD et al., 2006). Bager et al. (2010) estudaram uma população de T. dorbigni no sul do Rio Grande do Sul e obtiveram uma razão sexual equivalente a $1: 1$, utilizando apenas mergulho livre para a captura dos indivíduos. Outra população de T. dorbigni, estudada em um ambiente poluído no município de Pelotas (RS) e capturada com dois modelos de armadilhas, também não apresentou desvio de 1:1 (FAGUNDES et al., 2010). A razão sexual encontrada no presente estudo é semelhante a razão encontrada por Bujes et al. (2011), onde os autores utilizaram armadilhas "box trap" (similares às aqui utilizadas) além do método de busca ativa. O método de amostragem utilizado no presente estudo não parece ser responsável pela proporção entre os sexos, uma vez que permitiu a captura de indivíduos de distintas classes de tamanho. Entretanto, a análise de uma amostra maior da população será necessária para afirmar que a razão sexual encontrada neste trabalho reflete a estrutura da população na natureza, pois em

FIGURA 1: Comprimento máximo da carapaça (em mm) de indivíduos adultos de Trachemys dorbigni, capturados em São Gabriel, Rio Grande do Sul, Brasil. Barras cinzas - machos; Barras pretas - fêmeas.

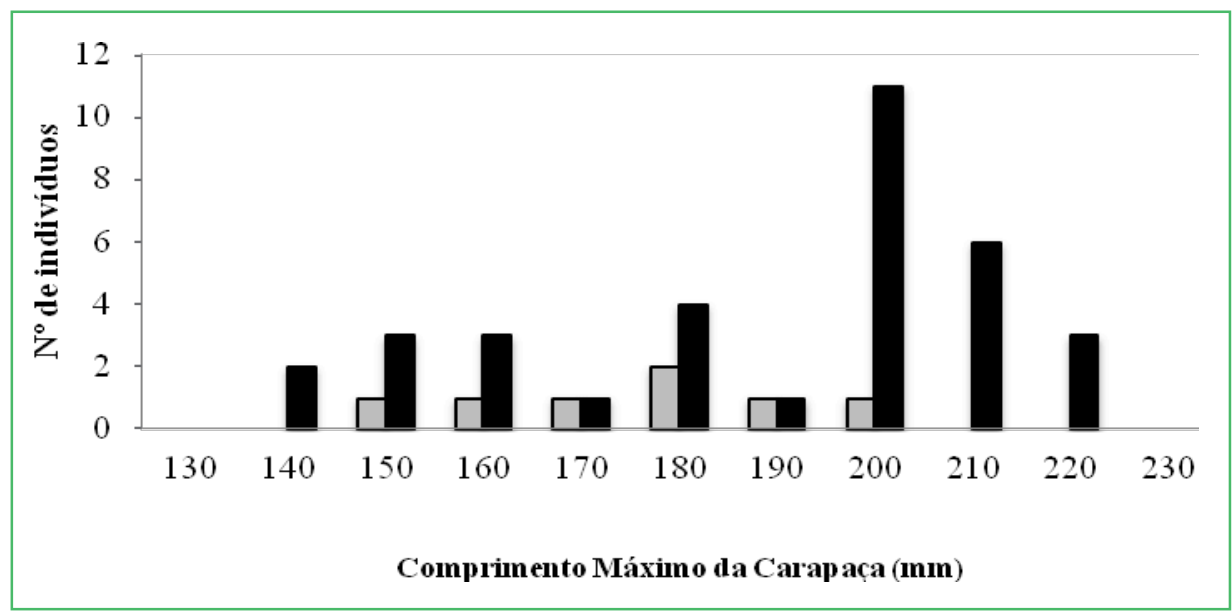


amostras pequenas a razão sexual encontrada pode ser fruto de uma amostragem seletiva (BURY, 1979).

A distribuição de frequência em classes de tamanho de CMC mostrou ser unimodal para machos e fêmeas. Seguindo o mesmo padrão encontrado em outras populações de T. dorbigni (BAGER et al., 2010; FAGUNDES et al., 2010; BUJES et al., 2011), este padrão unimodal também é valido para populações de Trachemys scripta (GIBBONS; GREENE, 1990; LOVICH et al., 1990). O padrão unimodal verificado neste estudo reforça o padrão de distribuição de classes de tamanho encontrado para populações do gênero Trachemys. A tendência á bimodalidade já foi observada em quelônios, como em machos e fêmeas de Podocnemis unifilis (FACHÍN-TERÁN; VOGT, 2004) e em fêmeas de $P$. sextuberculata (FACHÍN-TERÁN et al., 2003). Segundo Fachín-Terán et al. (2003) o padrão bimodal pode refletir o crescimento mais lento durante uma etapa da vida do animal.

Indivíduos de $T$. dorbigni estudados por vários autores mostraram-se maiores que os encontrados neste estudo, principalmente as fêmeas (FREIBERG, 1969; VANZOLINI, 1997; BUJES; VERRASTRO, 2007; BAGER et al., 2010; FAGUNDES et al., 2010; BUJES et al., 2011). Diferenças de tamanho corporal (CMC) entre populações podem ocorrer devido à quantidade e a qualidade de alimento disponível no ambiente, já que esse recurso determina os níveis de crescimento de um indivíduo (GIBBONS, 1967). O fato da população de São Gabriel viver em um ambiente aquático restrito (lagoa permanente), com pouca vegetação e sem locais apropriados para o assoalhamento aéreo, provavelmente tem influência no crescimento corpóreo dos animais. Os demais estudos com a espécie ocorreram em corpos d'água com dimensões maiores e características de habitat diferenciadas. De acordo com Dunham e Gibbons (1990) diferentes ambientes irão apresentar diferentes recursos alimentares, desta forma podemos inferir que o recurso alimentar disponível no ambiente seria o responsável pelos diferentes tamanhos corpóreos entre as populações de Trachemys dorbigni.

Os resultados obtidos mostraram fêmeas maiores do que os machos em seis medidas (ALT, CSMV, CMP, LMP, CCC e Peso) e menores em uma medida,
CCD1. Essas relações foram observadas em outras populações de T. dorbigni, onde foram encontradas fêmeas apresentando CCD1 menor que os machos (BUJES et al., 2011) e sendo maiores que estes paras as medidas de ALT, CMP e Peso (BAGER et al., 2010; FAGUNDES et al., 2010). Diferenças de tamanho entre machos e fêmeas provavelmente indicam diferenças ecológicas, fisiológicas e evolutivas entre os sexos (DUNHAM; GIBBONS, 1990). A vantagem das fêmeas atingirem maiores dimensões em relação aos machos está associada ao aumento do potencial reprodutivo (tamanho da ninhada), podendo dar origem a um maior número de descendentes (LOVICH; GIBBONS, 1992). Já os machos de espécies aquáticas, geralmente, são menores que as fêmeas, onde o menor tamanho dos machos aumenta a sua mobilidade, o que auxilia na localização das fêmeas (BERRY; SHINE, 1980).

Como já relatado anteriormente, as fêmeas mostraram-se mais pesadas que os machos, apresentando maior variação de peso. É sugerido por Bager et al. (2010) que a grande diferença de peso entre machos e fêmeas pode estar relacionada com a presença de ovos ou com a retenção de líquido pelas fêmeas, hipótese que condiz com os resultados obtidos para a população de São Gabriel. Há uma diferença significativa entre os pesos de machos e fêmeas, mas não entre os comprimentos de carapaça, portanto, a diferença em peso parece não estar relacionada com o tamanho do indivíduo, podendo estar associada a fatores reprodutivos.

A maioria das tartarugas de água doce apresenta algum grau de dimorfismo sexual (GIBBONS; GREENE, 1990). As diferenças geralmente são mais visíveis nos machos e são expressas por características secundárias como a melanização que surge no início da maturidade sexual e é realçada com o passar da idade (DUNHAM et al., 1988). Foi encontrado por Bager et al. (2010) que o VAO é uma característica importante na diferenciação entre machos e fêmeas de T. dorbigni. O presente estudo corrobora esta observação e também a encontrada por Cabrera (1998), que enfatiza a importância do CCD1 como variável que evidencia o dimorfismo sexual nessa espécie. É relatado que o dimorfismo nessa característica é fruto de adaptação dos machos para a cópula (MOLL, 1980; VOGT, 1980). Outra medida importante com 
relação ao dimorfismo sexual, a CCC, mostrou que as fêmeas apresentam a carapaça mais curvilínea que a dos machos. A CCC pode ter uma influência na fecundidade, assim como indicado para Testudo graeca graeca (KADDOUR et al., 2008), possibilitando um maior volume interno para acomodação dos ovos.

\section{Agradecimentos}

Agradecemos aos revisores anônimos pelas relevantes sugestões ao manuscrito. Ao Conselho Nacional de Desenvolvimento Científico e Tecnológico (CNPq) (484475/2008-0) pelo financiamento concedido durante o estudo. Ao IBAMA pelo fornecimento da licença de trabalho (14596-1/528136).

\section{Referências}

APONTE, C.; BARRETO, G. R.; TERBORGH, J. Consequences of habitat fragmentation on age structure and life history in a tortoise population. Biotropica, Maiden, v. 35, p. 550-555, 2003.

BAGER, A.; FREITAS, T. R. O.; KRAUSE, L. Morphological characterization of adults of Orbigny's slider Trachemys dorbignyi (Duméril \& Bibron 1835) (Testudines Emydidae) in southern Brazil. Tropical Zoology, Firenze, v. 23, p. 181-194, 2010.

BAGER, A.; ROSADO, J. L. O. Estimation of core terrestrial habitats for freshwater turtles in Southern Brazil based on nesting areas. Journal of Herpetology, Salt Lake City, v. 44, p. 658-662, 2010.

BATISTEllA, A. M.; CASTRO, C. P.; VOGT, R. C. Geographic Distribution, Trachemys adiutrix. Herpetological Review, Salt Lake City, v. 39, p. 107-108, 2008.

BERRY, J. F.; SHINE, R. Sexual size dimorphism and sexual selection in turtles (Order Testudines). Oecologia, Heidelberg, v. 44, p. 185-191, 1980.

BUJES, C. S.; MOLINA, F. N.; VERRASTRO, L. Population characteristics of Trachemys dorbigni (Testudines, Emydidae) from delta do Jacuí state park, Rio Grande do Sul, Southern Brazil. South American Journal of Herpetology, São Paulo, v. 6, p. 2734, 2011 .

BUJES, C. S.; VERRASTRO, L. Supernumerary epidermal shields and carapace variation in Orbigny's slider turtles, Trachemys dorbigni (Testudines, Emydidae). Revista Brasileira de Zoologia, Curitiba, v. 24, p. 666-672, 2007.

BUJES, C. S.; VERRASTRO, L. Chelonians from the Delta of Jacuí River, RS, Brazil: habitats use and conservation. Natureza e Conservação, Curitiba, v. 6, p. 157-170, 2008.

BURY, R. B. Population ecology of freshwater turtle. In: HURLLES, M.; MORLOCK, H. (Ed.). Turtles - perspective and research. New York: John Wiley \& Sons, 1979. p. 571-602.
CABRERA, M. R. Las tortugas continentales de Sudamérica Austral. Córdoba: Talleres gráficos BR Copias, 1998. 108 p.

CAGLE, F. R. A system of marking turtles for future identification. Copeia, Lawrence, v. 3, p. 170-173, 1939.

DODD JR, C. K. Secondary sex ratio variation among populations of the flattened musk turtle, Sternotherus depressus. Copeia, Lawrence, v. 4, p. 1041-1045, 1989.

DODD, K. L.; MURDOCK, C.; WIBBELS, T. Interclutch variation in sex ratios produced at pivotal temperature in the red-eared slider, a turtle with temperature-dependent sex determination. Journal of Herpetology, Salt Lake City, v. 40, p. 544-549. 2006.

DUNHAM, A. E.; GIBBONS, J. W. Growth of the slider turtle. In: GIBBONS, J. W. (Ed.). Life History and Ecology of the Slider Turtle. Washington: Smithsonian Institution Press, 1990. p. 135145.

DUNHAM, A. E.; MORIN, P. J.; WILBUR, H. M. Methods for the study of reptile populations. Biology of the Reptilia, Chicago, v. 16, p. 331-386, 1988.

FACHÍN-TERÁN, A.; VOGT, R. C.; THORBJARNARSON, J. B. Estrutura populacional, razão sexual e abundância de Podocnemis sextuberculata (Testudines, Podocnemididae) na Reserva de Desenvolvimento Sustentável Mamirauá, Amazonas, Brasil. Phyllomedusa, Belo Horizonte, v. 2, p. 43-63, 2003.

FACHÍN-TERÁN, A.; VOGT, R. C. Estrutura populacional, tamanho e razão sexual de Podocnemis unifilis (Testudines, Podocnemididae) no rio Guaporé (RO), norte do Brasil. Phyllomedusa, Belo Horizonte, v. 3, p. 29-42, 2004.

FAGUNDES, C. K.; BAGER, A.; CECHIN, S. T. Z. Trachemys dorbigni in an anthropic environment in southern Brazil: I) Sexual size dimorphism and population estimates. Herpetological Journal, Montrose, v. 20, p. 185-193, 2010.

FREIBERG, M. A. Una nueva subespecie de Pseudemys dorbigni (Duméril \& Bibron) (Reptilia, Testudines, Emydidae). Physis, Rio de Janeiro, v. 28, p. 299-314, 1969.

FRITZ, U.; HAVA, P. Checklist of chelonians of the world. Vertebrate Zoology, Dresden, v. 57, p. 149-368, 2007.

GIBBONS, J. W. Variation in growth rates in three populations of the painted turtle, Chrysemys picta. American Midland Naturalist, Notre Dame, v. 79, p. 517-519, 1967.

GIBBONS, J. W. Sex rations and their significance among turtle populations. In: GIBBONS, J. W. (Ed.). Life History and Ecology of the Slider Turtle. Washington: Smithsonian Institution Press, 1990. p. 171-182.

GIBBONS, J. W.; GREENE, J. L. Reproduction in the Slider and other species of turtles. In: GIBBONS, J. W. (Ed.). Life History and Ecology of the Slider Turtle. Washington: Smithsonian Institution Press, 1990. p. 124-134.

GIBBONS, J. W.; LOVICH, J. E.; TUCKER, A. D.; FITZSIMMONS, N. N.; GREENE, J. L. Demographic and ecological factors affecting conservation and management of the Diamondback Terrapin (Malaclemys terrapin) in South Carolina. Chelonian Conservation and Biology, Kansas, v. 4, p. 66-74, 2001.

KADDOUR, K. B.; EL MOUDEN, E. H.; SLIMANI, T.; BONNET, X.; LAGARDE, F. Sexual dimorphism in the greek tortoise: a 
test of the body shape hypothesis. Chelonian Conservation and Biology, Kansas, v. 7, p. 21-27, 2008.

LOVICH, J. E.; MCCOY, C. J.; GARSTKA, W. R. The development and significance of melanism in the slider turtle. In: GIBBONS, J. W. (Ed). Life History and Ecology of the Slider Turtle. Washington: Smithsonian Institution Press, 1990. p. 233-254.

LOVICH, J. E.; GIBBONS, J. W. A review of techniques for quantifying sexual size dimorphism. Growth, Development \& Aging, Bar Harbor, v. 56, p. 269-281, 1992.

MALUF, J. R. T. Nova classificação climática do Estado do Rio Grande do Sul. Revista Brasileira de Agrometeorologia, Santa Maria, v. 8, p. 141-150, 2000.

MOLINA, F. B.; GOMES, N. Incubação artificial dos ovos e processo de eclosão em Trachemys dorbigni (Duméril \& Bibron, 1835) (Reptilia, Testudines Emydidae). Revista Brasileira de Zoologia, Curitiba, v. 15, p. 135-143, 1998.

MOLL, E. O. Natural history of the river terrapin, Batagur baska (Gray) in Malaysia (TESTUDINES: EMYDIDAE). Malaysian Journal of Science, Kuala Lumpur, v. 6, p. 23-62, 1980.

PEREIRA, F. E.; DIEFENBACH, C. O. Growth in Trachemys dorbigni (Testudines, Emydidae). Biociências, Porto Alegre, v. 9, p. 21-31, 2001.
SEIDEL, M. E. Taxonomic observations on extant species and subespecies of slider turtles, genus Trachemys. Journal of Herpetology, Salt Lake City, v. 36, p. 258-292, 2002.

VANZOLINI, P. E. A new species of turtle, genus Trachemys, from state of Maranhão, Brasil (Testudines, Emydidae). Revista Brasileira de Biologia, São Carlos, v. 55, p. 111-125, 1995.

VANZOLINI, P. E. A note on the reproduction of Trachemys dorbignyi (Testudines, Emydidae). Revista Brasileira de Biologia, São Carlos, v. 57, p. 165-175, 1997.

VOGT, R. C. Natural history of the map turtles Graptemys pseudogeografica and $G$. ouachitensis in Wisconsin. Tulane Studies in Zoology and Botany, Belle Chasse, v. 22, p. 17-48, 1980.

WIBBELS, T.; COWAN, J.; LEBOEUF, R. Temperature-dependent sex determination in the red-eared slider turtle, Trachemys scripta. Journal of Experimental Zoology, Philadelphia, v. 281, p. 409-416, 1998. 